\title{
Horizontal Approximate Deconvolution for Stratified Flows: Analysis and Computations
}

\author{
Luigi C. Berselli ${ }^{1}$, Traian Iliescu ${ }^{2}$, and Tamay Özgökmen ${ }^{3}$ \\ 1 Dipartimento di Matematica Applicata "U. Dini", Via F. Buonarroti 1/c, \\ I-56127, Pisa ITALY, Phone:+390502213846, Fax: +390502213802, \\ berselli@dma.unipi.it \\ 2 Department of Mathematics, Virginia Tech, Blacksburg, VA, United States, \\ iliescu@vt.edu \\ 3 RSMAS/MPO, University of Miami, Miami, FL, United States, \\ tozgokmen@rsmas.miami.edu
}

Summary. In this paper we propose a new Large Eddy Simulation model derived by approximate deconvolution obtained by means of wave-number asymptotic expansions. This LES model is designed for oceanic flows and in particular to simulate mixing of fluids with different temperatures, density or salinity. The model -which exploits some ideas well diffused in the community- is based on a suitable horizontal filtering of the equations. We prove some a-priori estimates, showing certain mathematical properties and we present also the results of some preliminary numerical experiments.

Key words: Stratified flows, Rational/Clark LES model, Boussinesq equations, LES in domains with boundary

\section{Introduction}

Mixing is one of the most important processes to understand transport of pollutants as well as the details of thermohaline circulation. Despite the increase in computational power, the scales in the ocean circulation can not be all resolved simultaneously. Basin models are configured for $\mathcal{O}(1000 \mathrm{~km})$ to $\mathcal{O}(10 \mathrm{~km})$, and regional or coastal models from $\mathcal{O}(100 \mathrm{~km})$ to $\mathcal{O}(1 \mathrm{~km})$, requiring both sub-grid-scale parametrization. However, there exist small-scale ocean flows (which take place below this inherently coarse numerical resolution) that often play a significant role in an accurate representation of the large ocean scales. More precise motivations for the study of this physical problem and the requirement of suitable numerical methods to handle all scales is explained for instance in $[26,24]$. The mixing phenomena can be described mathematically by means of the Boussinesq system of partial differential equations: 


$$
\begin{aligned}
\partial_{t} \mathbf{u}+(\mathbf{u} \cdot \nabla) \mathbf{u}-\frac{1}{R e} \Delta \mathbf{u}+\nabla p & =-\frac{1}{F r^{2}} \rho^{\prime} \mathbf{e}_{3}, \quad \text { with } \nabla \cdot \mathbf{u}=0 \\
\partial_{t} \rho^{\prime}+(\mathbf{u} \cdot \nabla) \rho^{\prime}-\frac{1}{\operatorname{Re} \operatorname{Pr}} \Delta \rho^{\prime} & =0 .
\end{aligned}
$$

The unknowns $\left(\mathbf{u}, p, \rho^{\prime}\right)$ are velocity, pressure, and "salinity perturbation," respectively and $\mathbf{e}_{3}=(0,0,1)$. The non-dimensional parameters are the Reynolds number Re, the Prandtl number $\mathrm{Pr}$, and the Froude number Fr. The problem we consider takes naturally place in an elongated domain (We model the small portion of the ocean we consider as a parallelepiped)

$$
D:=\left\{x \in \mathbb{R}^{3}:-\pi<x_{1}, x_{2}<\pi, \quad-d<x_{3}<d\right\} \quad \text { with } \quad d<<1,
$$

hence the boundary is flat and we enforce periodic boundary conditions on the "horizontal variables" $x_{h}:=\left(x_{1}, x_{2}\right)$. We use the subscript " $h$ " to denote differential operators acting only on the horizontal variables. In particular, we use the following notation

$$
\Delta_{h}:=\partial_{x_{1}}^{2}+\partial_{x_{2}}^{2} \quad \text { and } \quad \nabla_{h}:=\left(\partial_{x_{1}}, \partial_{x_{2}}\right) .
$$

One of the features (which is well-know by practitioners) of this problem is that filtering seems to be required only in the horizontal directions, because the mixing takes place mainly along these two directions. In a previous work [3] we started analyzing it by means of stochastic parametrization and Itô's calculus. Since the problem we have in mind is that of stratified fluids, another justification for the use of horizontal viscosities/filtering comes from the study of Ekman boundary layers for rotating fluids (see [9] and references therein) and in particular the system of the Navier-Stokes equations with partial viscosity:

$$
\partial_{t} \mathbf{u}+(\mathbf{u} \cdot \nabla) \mathbf{u}-\frac{1}{R e} \Delta_{h} \mathbf{u}+\nabla p=\mathbf{f}, \quad \text { with } \nabla \cdot \mathbf{u}=0 .
$$

These are the main modeling motivations for the anisotropic LES method (2) we will introduce. Other motivations, which are consequences of the mathematical properties, will be explained in the sequel, but another main feature of the new method we propose is that we do not need artificial boundary conditions. The objective of the present paper is to propose a new LES method, to prove existence of suitable classes of solutions, and to compare the results of some 3D simulations performed with other LES methods.

\section{An anisotropic Large Eddy Simulation model}

One of the earliest LES models is the "Gradient" method (known also as Taylor or Clark method and introduced in [21, 10]) which is based on an 
approximation of the subgrid scale term by means of asymptotic expansion in wave-numbers. In the Gradient model the turbulent stress-tensor is $\boldsymbol{\tau}_{\mathrm{G}}(\mathbf{w}, \mathbf{w})=\alpha^{2} \nabla \mathbf{w} \nabla \mathbf{w}^{T}$, where $\left[\nabla \mathbf{w} \nabla \mathbf{w}^{T}\right]_{i j}:=\sum_{k=1}^{3} \partial_{x_{k}} w^{i} \partial_{x_{k}} w^{j}$. The derivation of this model and the basic results regarding the mathematical analysis are collected in [5]. It is well-known that instabilities occur in the numerical implementation and that some kind of smoothing must be added in order to have effective simulations, see [17]. In particular, a filtered version of the Gradient method is called Rational or ${ }^{4}$ Clark- $\alpha$ method, and the stress-tensor reads as

$$
\boldsymbol{\tau}_{\mathrm{R}-\mathrm{C}}(\mathbf{w}, \mathbf{w})=\left(\mathrm{I}-\alpha^{2} \Delta\right)^{-1} \alpha^{2} \nabla \mathbf{w} \nabla \mathbf{w}^{T},
$$

cf. [34, 33, 31, 32]. The Rational method has been derived [16] by a rational approximation of wave-numbers, while the Clark- $\alpha$ method is based on analogies with the Helmholtz filtering. The mathematical analysis of these models can be found in $[8,4]$. Following the approach described in the introduction we consider a smoothing acting only in the horizontal variables, which can be performed by means of convolution with the following kernel: $g_{\alpha}(x)=\frac{1}{\pi \alpha^{2}} \operatorname{Exp}\left(-\frac{x_{1}^{2}+x_{2}^{2}}{\alpha^{2}}\right)$. By taking the 2D Fourier Transform, by performing the Taylor series expansion, and by neglecting terms which are formally of order of $\alpha^{4}$ one gets (apart multiplicative constants) the following expression for the subgrid scale term $\boldsymbol{\tau}_{\mathrm{hG}}(\mathbf{w}, \mathbf{w})=\alpha^{2} \nabla_{h} \mathbf{w} \nabla_{h} \mathbf{w}^{T}$, where $\left[\nabla_{h} \mathbf{w} \nabla_{h} \mathbf{w}^{T}\right]_{i j}:=\alpha^{2} \sum_{k=1}^{2} \partial_{x_{k}} w^{i} \partial_{x_{k}} w^{j}$. We mainly consider the "horizontal version" of the Rational-Clark- $\alpha$ method,

$$
\boldsymbol{\tau}_{\mathrm{hR-C}}(\mathbf{w}, \mathbf{w})=\left(\mathrm{I}-\alpha^{2} \Delta_{h}\right)^{-1} \alpha^{2} \nabla_{h} \mathbf{w} \nabla_{h} \mathbf{w}^{T} .
$$

From the computational point of view the inversion of a horizontal Laplacian is less time/memory consuming than the usual one, but the main advantage of the use of the horizontal Laplacian is that there is no need to introduce extra/artificial boundary conditions for the Helmholtz operator and that the value $\mathbf{w}$ on the boundary of the domain $D$ can be imposed. The issue of the boundary conditions for LES models is generally very complex, cf. [5, Ch.9], and the challenging property of our approach based on horizontal filtering is that part of the problem (due also to the particular shape of the domain) is overcome. In the sequel, we sketch the main mathematical properties of the space filtered Navier-Stokes equations approximated by the "horizontal Rational/Clark" model

$$
\partial_{t} \mathbf{w}+(\mathbf{w} \cdot \nabla) \mathbf{w}-\frac{1}{R e} \Delta \mathbf{w}+\nabla \cdot\left(\mathrm{I}-\alpha^{2} \Delta_{h}\right)^{-1} \alpha^{2} \nabla_{h} \mathbf{w} \nabla_{h} \mathbf{w}^{T}+\nabla q=\mathbf{f},
$$

with $\nabla \cdot \mathbf{w}=0$. Full details on the horizontal filtering, in conjunction with other LES models (with more appealing and neat mathematical properties) will appear in [2].

\footnotetext{
${ }^{4}$ The two methods differ by a multiplicative factor, which is nevertheless critical for the well-posedness results, see Remark 2 and also Section 5.
} 


\subsection{Some properties of the horizontal Rational/Clark model}

Given a vector field $\mathbf{f}$, the averaged field $\left(\mathrm{I}-\alpha^{2} \Delta_{h}\right)^{-1} \mathbf{f}$ is defined by solving the following horizontal Helmholtz-Stokes problem:

$$
\begin{aligned}
\mathbf{u}-\alpha^{2} \Delta_{h} \mathbf{u}+\nabla q & =\mathbf{f}, \quad \text { with } \nabla \cdot \mathbf{u}=0 \text { in } D, \\
\mathbf{u} \cdot \mathbf{n} & =0 \quad \text { on } \partial D,
\end{aligned}
$$

and throughout the paper $\alpha>0$ will be a fixed number. We use the notation

$$
L^{2}(D):=\left\{\phi: D \rightarrow \mathbb{R}, x_{h} \text {-periodic, measurable, with } \int_{D}|\phi|^{2} d x<+\infty\right\}
$$

and $\mathbb{L}^{2}:=\left(L^{2}(D)\right)^{3}$. We use the same symbol $\|$. $\|$ for the norm in both spaces. Next, by setting $\partial D:=\left\{x \in \mathbb{R}^{3}:-\pi<x_{1}, x_{2}<\pi, x_{3}= \pm d\right\}$ we can define

$\mathbb{H}_{h}^{1}:=\left\{\mathbf{u} \in L^{2}: \nabla_{h} \mathbf{u} \in\left(L^{2}(D)\right)^{6}, \nabla \cdot \mathbf{u} \in L^{2}(D), \mathbf{u} \cdot \mathbf{n}=0\right.$ in $\left.H^{-1 / 2}(\partial D)\right\}$,

where $\mathbf{n}$ denotes the exterior unit normal vector on $\partial D$. Observe also that since $\mathbf{u}$ has divergence in $L^{2}(D)$, then $\frac{\partial u^{3}}{\partial x_{3}}=-\nabla_{h} \cdot \mathbf{u}_{h}=-\frac{\partial u^{1}}{\partial x_{1}}-\frac{\partial u^{2}}{\partial x_{2}} \in L^{2}(D)$. This shows that some extra-regularity for the vertical component of the velocity is obtained for free and this another interesting mathematical advantage.

Lemma 1 (See [2]). Let $\mathbf{f} \in \mathbb{L}^{2}$ and $\alpha>0$. Then, there exists a unique $(\mathbf{u}, q) \in \mathbb{H}_{h}^{1} \times L^{2}(D) / \mathbb{R}$ solution of $(3)$ and a constant $c=c(\alpha)$ such that

$$
\|\mathbf{u}\|+\alpha\left\|\nabla_{h} \mathbf{u}\right\|+\alpha\left\|\nabla u^{3}\right\|+\|q\| \leq c\|\mathbf{f}\|,
$$

Remark 1. A relevant point (for a rigorous proof see again [2]) is that one can prove that $q=0$. Consequently one can solve three uncoupled Helmholtz problem, instead of the Stokes problem. This motivates the name HelmholtzStokes.

What is most important for understanding the mathematical properties of solutions of (2) is the following lemma.

Lemma 2. For all sufficiently smooth vector fields $\mathbf{w}$ on $D, x_{h}$-periodic, such that $\nabla \cdot \mathbf{w}=0$ in $D$ and $\mathbf{w} \cdot \mathbf{n}=0$ on $\partial D$, it holds

$$
\int_{D}\left[(\mathbf{w} \cdot \nabla) \mathbf{w}+\nabla \cdot\left(\mathrm{I}-\alpha^{2} \Delta_{h}\right)^{-1} \alpha^{2} \nabla_{h} \mathbf{w} \nabla_{h} \mathbf{w}^{T}\right] \cdot\left(\mathrm{I}-\alpha^{2} \Delta_{h}\right) \mathbf{w} d x=0 .
$$

Proof. The proof is obtained by direct integration by parts (cf. [8, 4]). In fact, as usual $\int_{D}(\mathbf{w} \cdot \nabla) \mathbf{w} \cdot \mathbf{w} d x=0$ and next, observe that

$$
\begin{aligned}
\int_{D}(\mathbf{w} \cdot \nabla) \mathbf{w} \cdot\left(-\alpha^{2} \Delta_{h} \mathbf{w}\right) & =\alpha^{2} \sum_{k=1}^{2} \int_{D} \partial_{x_{k}}[(\mathbf{w} \cdot \nabla) \mathbf{w}]\left(\partial_{x_{k}} \mathbf{w}\right) \\
& =\alpha^{2} \sum_{i, j=1}^{3} \sum_{k=1}^{2} \int_{D} \partial_{x_{k}} \mathbf{w}^{j} \partial_{x_{j}} \mathbf{w}^{i} \partial_{x_{k}} \mathbf{w}^{i} d x
\end{aligned}
$$


since one term vanishes by integration by parts. Moreover, we have

$\int_{D} \nabla \cdot\left(\mathrm{I}-\alpha^{2} \Delta_{h}\right)^{-1} \alpha^{2} \nabla_{h} \mathbf{w} \nabla_{h} \mathbf{w}^{T} \cdot\left(\mathrm{I}-\alpha^{2} \Delta_{h}\right) \mathbf{w}=\alpha^{2} \int_{D} \nabla \cdot\left(\nabla_{h} \mathbf{w} \nabla_{h} \mathbf{w}^{T}\right) \mathbf{w}$,

since in our setting the operator $\left(\mathrm{I}-\alpha^{2} \Delta_{h}\right)$ is self-adjoint. Finally, a further integration by parts (possible since $\mathbf{w} \cdot \mathbf{n}=0$ on $\partial D$ ) shows that

$$
\alpha^{2} \int_{D} \nabla \cdot\left(\nabla_{h} \mathbf{w} \nabla_{h} \mathbf{w}^{T}\right) \mathbf{w}=-\alpha^{2} \int_{D} \sum_{i, j=1}^{3} \sum_{k=1}^{2} \partial_{x_{k}} \mathbf{w}^{i} \partial_{x_{k}} \mathbf{w}^{j} \partial_{x_{j}} \mathbf{w}^{i},
$$

and adding together the various terms we end the proof.

Remark 2. Lemma 2 shows that $\left(\mathrm{I}-\alpha^{2} \Delta_{h}\right) \mathrm{w}$ is a "good multiplier" but again it is relevant (concerning the boundary conditions) that it involves only derivatives in the horizontal directions. Moreover, to be precise, it is important to note that the Rational model corresponds to the following stress-tensor

$$
\boldsymbol{\tau}_{\mathrm{RLES}}(\mathbf{w}, \mathbf{w})=\left(\mathrm{I}-\frac{\alpha^{2}}{2} \Delta\right)^{-1} \alpha^{2} \nabla \mathbf{w} \nabla \mathbf{w}^{T}
$$

In this case the "good multiplier" is $\left(\mathrm{I}-\frac{\alpha^{2}}{2} \Delta\right) \mathrm{w}$ but the factor $\alpha^{2} / 2$ does not imply that the cancellation of the nonlinearities, as in Lemma 2. Results in $[4,1]$ show that the term $\nabla \cdot\left(\mathrm{I}-\frac{\alpha^{2}}{2} \Delta\right)^{-1} \alpha^{2} \nabla \mathbf{w} \nabla \mathbf{w}^{T}$ plays the same role (in terms of Sobolev spaces inequalities) of the convective term. This prevents from proving global-in-time estimates. This is one of the subtle differences between the Clark- $\alpha$ and the Rational Model. Obviously the same occurs also for the horizontal version of both methods.

The main result concerning system (2) is the following.

Theorem 1. Let be given $\mathbf{w}_{0} \in \mathbb{H}_{h}^{1}$, and $\mathbf{f} \in L^{2}\left(0, T ; L^{2}\right)$. Then, there exists a solution $\mathbf{w}\left(x_{h}\right.$-periodic and vanishing on $\left.\partial D\right)$ in the sense of distributions to system (2) such that

$$
\mathbf{w}, \nabla_{h} \mathbf{w}, \nabla w^{3} \in L^{\infty}\left(0, T ; L^{2}\right) \cap L^{2}\left(0, T ; H^{1}\right) .
$$

Proof. We sketch the proof, that is based on the usual Galerkin method. We look for an approximate solution $\mathbf{w}^{m}=\sum_{k=1}^{m} c^{k}(t) \mathcal{E}_{k}(x)$, where $\mathcal{E}_{k}(x)$ are eigenfunction of the Stokes operator with our boundary conditions. The explicit form of the eigenfunctions is know, see [29, 23], but for our purposes it is enough to observe that they are of the type $\mathrm{e}^{\mathrm{i}\left(k_{1} x_{1}+k_{2} x_{2}\right)} f\left(x_{3}\right)$, for $k_{1}, k_{2} \in \mathbb{Z}$ and for certain smooth $f$, vanishing at $x_{3}= \pm d$. This is relevant, since $\Delta_{h} \mathcal{E}_{k}$ still is an eigenfunction. Hence, the a priori estimate obtained by testing the equation by $\left(\mathrm{I}-\alpha^{2} \Delta_{h}\right) \mathbf{w}^{m}$ is completely justified. By performing standard integrations by parts (possible by $x_{h}$-periodicity) we get $\int_{D} \partial_{t} \mathbf{w}^{m} \cdot\left(\mathrm{I}-\alpha^{2} \Delta_{h}\right) \mathbf{w}^{m}=\frac{1}{2} \frac{d}{d t}\left(\left\|\mathbf{w}^{m}\right\|^{2}+\alpha^{2}\left\|\nabla_{h} \mathbf{w}^{m}\right\|^{2}\right)$ and also 
$-\int_{D} \Delta \mathbf{w}^{m} \cdot\left(\mathrm{I}-\alpha^{2} \Delta_{h}\right) \mathbf{w}^{m}=\left\|\nabla \mathbf{w}^{m}\right\|^{2}+\alpha^{2}\left\|\nabla \nabla_{h} \mathbf{w}^{m}\right\|^{2}$. Hence, application of Lemma 2 shows the following differential inequality for $\mathbf{w}^{m}$ :

$$
\frac{1}{2} \frac{d}{d t}\left(\left\|\mathbf{w}^{m}\right\|^{2}+\alpha^{2}\left\|\nabla_{h} \mathbf{w}^{m}\right\|^{2}\right)+\frac{1}{R e}\left(\left\|\nabla \mathbf{w}^{m}\right\|^{2}+\alpha^{2}\left\|\nabla \nabla_{h} \mathbf{w}^{m}\right\|^{2}\right) \leq c\|\mathbf{f}\|^{2} .
$$

Uniform bounds in $m \in \mathbb{N}$ and other standard arguments, see e.g.. [11], can be used to prove that (a sub-sequence of) $\mathbf{w}^{m}$ converges (as $m \rightarrow+\infty$ ) towards a solution of (2). Regularity of $w^{3}$ follows from $\nabla \cdot \mathbf{w}=0$.

Remark 3. The solution w has regularity properties which lie "in between" those of weak and strong solutions for the NSE (In particular we are missing the control of $\partial_{x_{3}} \mathbf{w}$ in $L^{\infty}\left(0, T ; L^{2}\right)$.) Consequently we are not able to prove uniqueness within this class of solutions. In addition, one cannot start a bootstrapping argument to improve the regularity of w: by using as test function $\left(\mathrm{I}-\alpha^{2} \Delta_{h}\right)^{2} \mathbf{w}^{m}$ (the counterpart of the $H^{2}$-estimates in [8]) one does not get good a priori estimates. Roughly speaking, it seems that multipliers which are good for $(\mathbf{w} \cdot \nabla) \mathbf{w}$ are not good for $\nabla \cdot\left(\mathrm{I}-\frac{\alpha^{2}}{2} \Delta\right)^{-1} \alpha^{2} \nabla_{h} \mathbf{w} \nabla_{h} \mathbf{w}^{T}$ and vice versa. Based on these observations we conjecture that this is not the best model (at least from the point of view of uniqueness and stability of solutions) to be implemented with the horizontal filtering. Much better theoretical results can be proved in the framework of Approximate Deconvolution Models (ADM), à la Stolz and Adams [30] and Layton Lewandowski [20] (cf. also [2]). With these models the special expression of the stress-tensor allow us to prove uniqueness using the same multiplier and just (4).

\section{On the Boussinesq system}

We consider now the Boussinesq system (1) and we study a LES model in which the velocity equation is filtered, while not the equation for the salinity. This because results of $[19,13]$ show that in presence of nonzero viscosity (and also with vanishing diffusivity) the classical conditions which ensure regularity of the NSE imply the continuation of smooth solutions of (1). Improved theoretical results on this topic will appear in a forthcoming paper [6]. Here, we consider the following horizontal LES model for the Boussinesq system (still with $\nabla \cdot \mathbf{w}=0$ )

$$
\begin{array}{r}
\partial_{t} \mathbf{w}+(\mathbf{w} \cdot \nabla) \mathbf{w}-\frac{1}{R e} \Delta \mathbf{w}+\nabla \cdot\left(\mathrm{I}-\alpha^{2} \Delta_{h}\right)^{-1} \alpha^{2} \nabla_{h} \mathbf{w} \nabla_{h} \mathbf{w}^{T} \\
+\nabla q=-\frac{1}{F r^{2}} \rho^{\prime} \mathbf{e}_{3} \\
\partial_{t} \rho^{\prime}+(\mathbf{w} \cdot \nabla) \rho^{\prime}-\frac{1}{\operatorname{RePr}} \Delta \rho^{\prime}=0 .
\end{array}
$$

With the same Galerkin approach of Sec. 2.1 one proves the following result. 
Theorem 2. Let be given $\left(\mathbf{w}_{0}, \rho_{0}^{\prime}\right) \in \mathbb{H}_{h}^{1} \times L^{2}$. Then, there exists a weak solution to $(5)$ ( $x_{h}$-periodic and with $\mathbf{w}$ vanishing on $\partial D$ ) such that

$$
\mathbf{w}, \nabla_{h} \mathbf{w}, \nabla w^{3}, \rho^{\prime} \in L^{\infty}\left(0, T ; L^{2}\right) \cap L^{2}\left(0, T ; H^{1}\right)
$$

The proof, is based on the following a-priori estimate (obtained by using as test function $\left(\left(\mathrm{I}-\alpha^{2} \Delta_{h}\right) \mathbf{w}, \rho^{\prime}\right)$ and by performing some integrations by parts)

$$
\begin{aligned}
\frac{1}{2} \frac{d}{d t}\left(\|\mathbf{w}\|^{2}+\alpha^{2}\left\|\nabla_{h} \mathbf{w}\right\|^{2}+\left\|\rho^{\prime}\right\|^{2}\right)+\frac{1}{R e}\left(\|\nabla \mathbf{w}\|^{2}\right. & \left.+\alpha^{2}\left\|\nabla \nabla_{h} \mathbf{w}\right\|^{2}\right) \\
& +\left\|\nabla \rho^{\prime}\right\|^{2} \leq c\left(\|\rho\|^{2}+\|\mathbf{f}\|^{2}\right),
\end{aligned}
$$

and by using the same standard tools as before. Also in this case we are not able to prove uniqueness, since the control of the whole $\nabla \mathbf{w}$ is missing.

In the next section we explain how the results can be improved by changing the class of models we consider. In particular, here we are testing the horizontal Rational/Clark method since it is the one for which we have at disposal a numerical code to perform preliminary assessment and we can precisely compare the results with those previously obtained. In addition, we wanted to test the performances of these methods in situations where the theoretical results are not conclusive.

\section{Perspectives for future studies}

Even if the numerical simulations for (2)-(5) are quite nice, the results are not completely satisfactory from the point of view of functional analysis. Good theoretical results can be surely obtained if the regularization in (5) is done with the Clark- $\alpha$ model instead of its horizontal counterpart, while less trivial is the limit $k:=\frac{1}{\operatorname{RePr}} \rightarrow 0^{+}$for this model.

Moving to ADM we can easily show that the following system is well-posed

$$
\begin{array}{r}
\partial_{t} \mathbf{w}+\nabla \cdot\left(\mathrm{I}-\alpha^{2} \Delta\right)^{-1}(\mathbf{w} \otimes \mathbf{w})-\frac{1}{R e} \Delta \mathbf{w}+\nabla q=-\frac{1}{F r^{2}} \rho^{\prime} \mathbf{e}_{3} . \\
\partial_{t} \rho^{\prime}+(\mathbf{w} \cdot \nabla) \rho^{\prime}-\frac{1}{R e P r} \Delta \rho^{\prime}=0 .
\end{array}
$$

This is the Boussinesq version of the Layton-Lewandowski model (or simplified Bardina) and good properties follow because with $\left(\left(\mathrm{I}-\alpha^{2} \Delta\right) \mathbf{w}, \rho^{\prime}\right)$ as test function, one gets directly $\nabla \mathbf{u} \in L^{\infty}\left(0, T ; L^{2}\right)$, cf. [20]. Observe that no regularization is needed in the equation for $\rho^{\prime}$. The computational problems with this model concern the boundary conditions, since the inversion of the Laplace operator requires $a d$ hoc boundary conditions on $\partial D$ (Essentially it works only in the periodic setting.) This observation is one of the main reasons that led to the introduction of the horizontal version of this model in [2] and for Boussinesq system, this will be naturally generalized in considering the equation 


$$
\partial_{t} \mathbf{w}+\nabla \cdot\left(\mathrm{I}-\alpha^{2} \Delta_{h}\right)^{-1}(\mathbf{w} \otimes \mathbf{w})-\frac{1}{R e} \Delta \mathbf{w}+\nabla q=-\frac{1}{F r^{2}} \rho^{\prime} \mathbf{e}_{3},
$$

coupled with $(6)_{2}$. From the mathematical point of view the resulting system does not give the sufficient control on $\nabla \mathbf{w}$ to prove uniqueness. Guided by the results on math analysis (by using the same tools as in [2] we can prove existence and uniqueness of strong solutions, globally in time) we conjecture that a suitable ADM model for Boussinesq will be the following

$$
\begin{array}{r}
\partial_{t} \mathbf{w}+\nabla \cdot\left(\mathrm{I}-\alpha^{2} \Delta_{h}\right)^{-1}(\mathbf{w} \otimes \mathbf{w})-\frac{1}{R e} \Delta \mathbf{w}+\nabla q=-\frac{1}{F r^{2}} \rho^{\prime} \mathbf{e}_{3}, \\
\partial_{t} \rho^{\prime}+\nabla \cdot\left(\mathrm{I}-\alpha^{2} \Delta_{h}\right)^{-1}\left(\rho^{\prime} \mathbf{w}\right)-\frac{1}{R e P r} \Delta \rho^{\prime}=0 .
\end{array}
$$

All theoretical results on the Boussinesq equations will be collected in the forthcoming paper [6], while testing of all different methods will be the object of future research.

\section{Numerical Results}

In this section, we investigate how the horizontal RLES model and the horizontal Clark- $\alpha$ models perform in the numerical simulation of $3 \mathrm{D}$ turbulent stratified flows. To this end, we compare the performance of four LES models: 1) the RLES model, 2) the Clark- $\alpha$ model, 3) the horizontal RLES model, and 4) the horizontal Clark- $\alpha$ model - against DNS results. The comparison criterion is simple: the closer the LES results are to the benchmark DNS results, the better the LES model. To ensure a fair assessment of the performance of the LES models, we also included under-resolved numerical simulations without any LES modeling, which we denoted by DNS*. Thus, it is expected that the LES models produce better results than the DNS*, at the very least.

Although most of the theoretical developments in this paper have been centered around the NSE (see also [2]), the numerical illustrations in this section are for the Boussinesq equations, since as explained in the introduction it is one of the commonly used mathematical models in the numerical investigation of oceanic and atmospheric flows. The model setup is similar to that in [25], which contains a detailed discussion of the boundary conditions, initial conditions and parameters used. We now briefly list them; for more details, the reader is referred to [25].

We consider the lock-exchange problem, a popular benchmark problem for the numerical investigation of mixing in stratified flows [26, 24, 25]. The computational domain is $-\frac{L}{2} \leq x \leq \frac{L}{2}, 0 \leq y \leq W$, and $0 \leq z \leq H$, where $L / H=2$ and $W / H=1$. At the top, bottom, left and right boundaries, noflow and free-slip boundary conditions are used for the velocity components $(u, v, w)$, while no-flux (insulation) conditions are used for the density perturbation $\rho^{\prime}$, i.e., $\frac{\partial w}{\partial \mathbf{n}}=0 ;(u, v, w) \cdot \mathbf{n}=0 ; \frac{\partial \rho^{\prime}}{\partial \mathbf{n}}=0$, where $\mathbf{n}$ is the normal to 
the boundary. In the horizontal directions, periodic boundary conditions are used both for velocity and density perturbation fields. $u(x, 0, z)=u(x, W, z)$; $v(x, 0, z)=v(x, W, z) ; \rho^{\prime}(x, 0, z)=\rho^{\prime}(x, W, z)$. Since $\alpha \rightarrow 0^{+}$near the boundary, the filtered variables approach the unfiltered variables. Thus, for the LES models, we used the same boundary conditions as those used for the DNS. The non-dimensional parameters have the same values as those in [25]: The Froude number is $F r=2^{-\frac{1}{2}}$, the Prandtl number is $\operatorname{Pr}=7$, and the Reynolds number is $R e=10^{4}$. The numerical study is conducted using Nek5000, which is a spectral element code developed by Paul Fischer and his group [27, 22, 14]. All experiments were run on Virginia Tech's SystemX, based on 2200 Apple G5 processors with $2.3 \mathrm{GHz}$ and InfiniBand interconnect. The accuracy of the LES models is evaluated through a posteriori testing. The main measure used is the background/reference potential energy (RPE), which exactly quantifies mixing in an enclosed system [25]. RPE is the minimum potential energy that can be obtained through an adiabatic redistribution of the water masses. Finally, for the RLES model, we chose $\gamma=3$ and $\gamma_{T}=15$, cf. [24, 25] for the notation.

There are also a couple of significant differences from the previous studies [24, 25]. First, in this study we are integrating much longer in time. As explained next, this has an effect on the conclusions regarding the performance of the LES models. Second, we implemented the horizontal version of the RLES and Clark- $\alpha$ models. Although the gradient tensor has been implemented in its horizontal form, the Helmholtz operator is still in its original isotropic form. We are currently implementing its anisotropic (horizontal) version.

The results of our numerical simulations are presented in figures 1, 2, and 3. In Figure 1, we present snapshots of DNS for the density perturbation $\rho^{\prime}$ at different times. This time evolution of the density perturbation will represent the benchmark for our LES runs.

In figure 2, we present snapshots of the density perturbation $\rho^{\prime}$ at $t=3.0$. Notice that all four models (horizontal RLES, horizontal Clark- $\alpha$, DNS, and DNS*) produce practically indistinguishable results.

Finally, in figure 3 we investigate the ability of the LES models to reproduce the DNS RPE curve. We also compare the isotropic and horizontal versions of the LES models. The behavior of the LES models depends on the time-interval considered. At the beginning of the numerical simulation, both LES models (horizontal RLES and horizontal Clark- $\alpha$ ) produce better results than the under-resolved simulation (DNS*) and the horizontal RLES model is more accurate than the Clark- $\alpha$ model. Towards the end of the simulation, however, the quality of the results produced by the LES models degrades. Indeed, the LES models yield RPE curves that are farther away from the benchmark RPE curve than the RPE curve produced by the under-resolved DNS* runs. We emphasize that this behavior was not displayed in our previous studies [24,25], since there we did not integrate as long in time as in our present study. 
These new results open a new research avenue that we are currently pursuing. On the other hand, the preliminary tests of this report show that the new methods we propose are promising and may be used to improve performances of previous simulations. Further analysis and tests are running, and we hope to give some new insight in the numerical simulation of mixing phenomena involved in ocean flows.
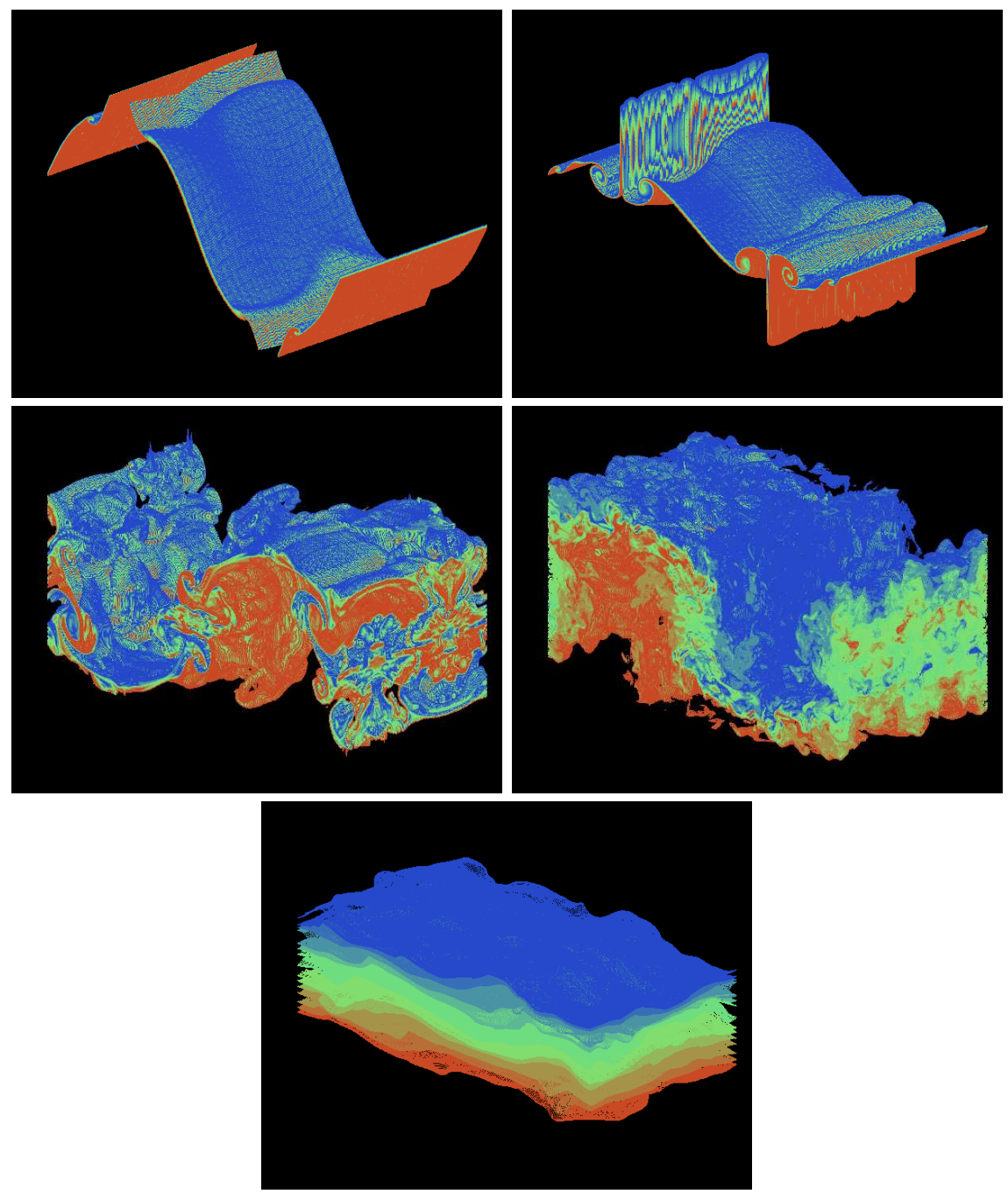

Fig. 1. Density perturbation snapshots. DNS at: (a) $\mathrm{t}=0.8$; (b) $\mathrm{t}=1.2 ;$ (c) $\mathrm{t}=3.0$; (d) $\mathrm{t}=5.0$; and (e) $\mathrm{t}=45.0$. 

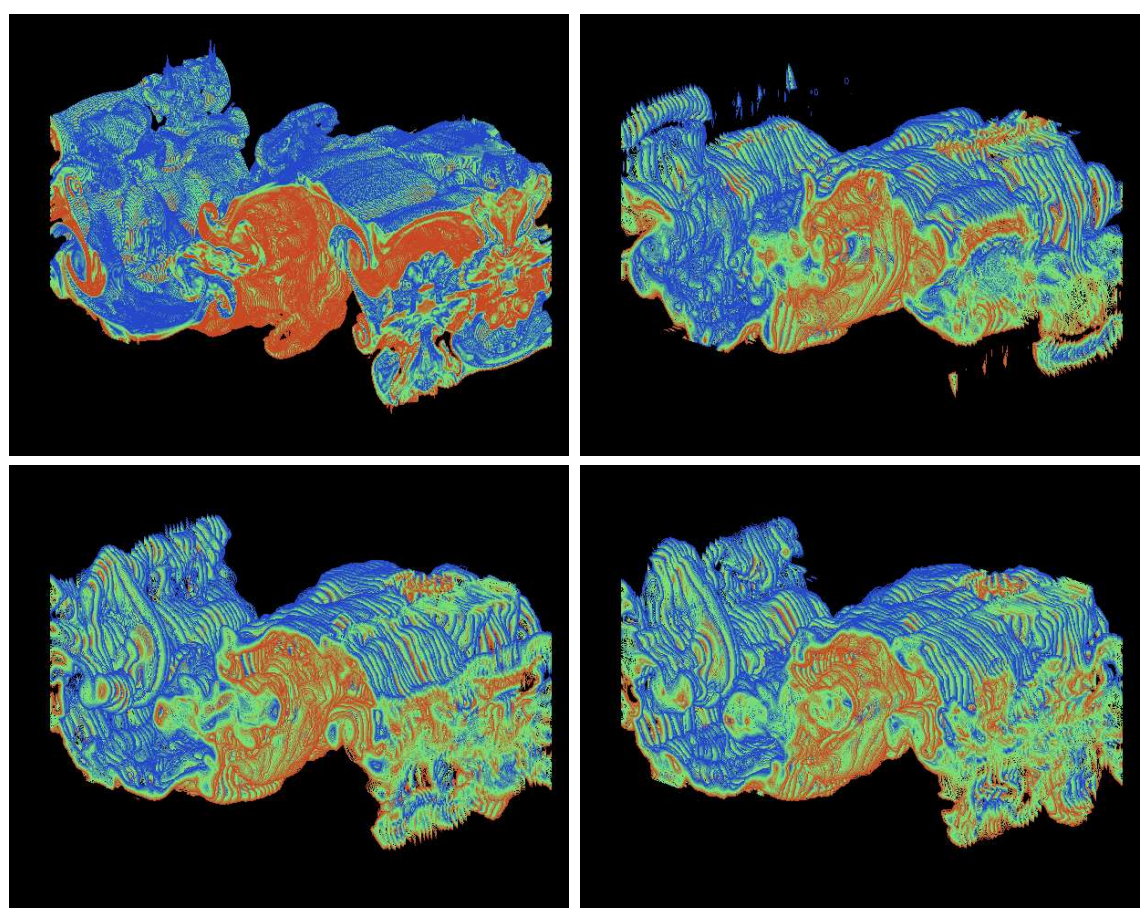

Fig. 2. Density perturbation snapshots. (a) DNS; (b) DNS*; (c) Clark- $\alpha$ horizontal; and (d) RLES horizontal.

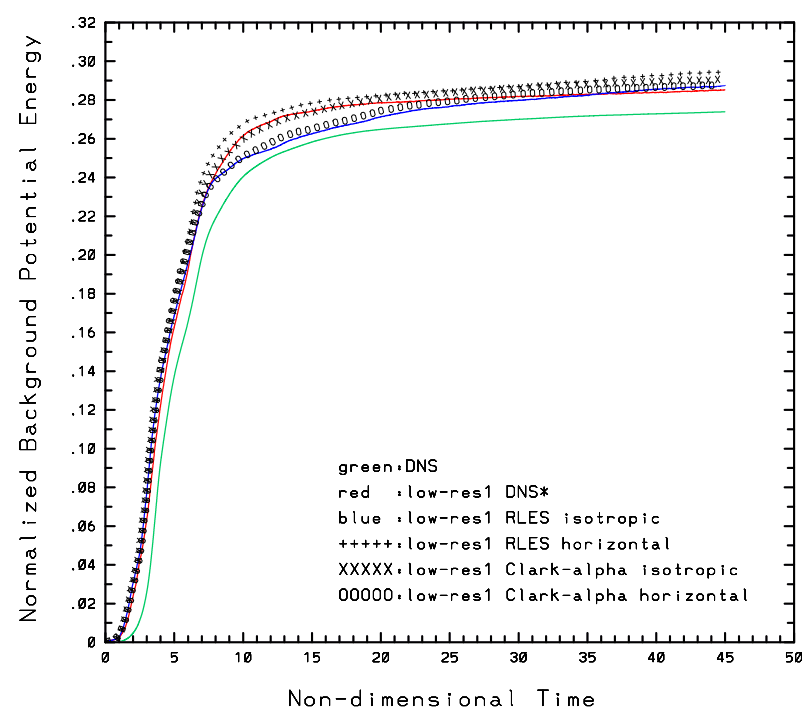

Fig. 3. RPE curves for DNS, DNS*, Clark- $\alpha$ horizontal, RLES horizontal, Clark- $\alpha$, and RLES. 


\section{References}

1. Barbato D, Berselli LC, Grisanti CR (2007) J. Math. Fluid Mech. 9:44-74

2. Berselli LC (2009) Analysis of a scale similarity LES model designed for certain stratified flows. Tech. Report., Univ. Pisa

3. Berselli LC, Flandoli F (2009) On a stochastic approach to Eddy Viscosity models for turbulent flows. In: Contributions to current challenges in mathematical fluid mechanics, Birkhäuser, Basel

4. Berselli LC, Galdi GP, Iliescu T, Layton WJ (2002) Math. Models Methods Appl. Sci. 12:1131-1152

5. Berselli LC, Iliescu T, Layton W J (2006) Mathematics of Large Eddy Simulation of turbulent flows. Springer-Verlag, Berlin

6. Berselli LC, Spirito S (2009) Remarks on the Boussinesq equations. In preparation

7. Cantero MI, Lee JR, Balachandar S, Garcia M (2007) J. Fluid Mech. 586:1-39

8. Cao C, Holm DD, Titi ES (2005) J. Turbul. 6, Paper 20.

9. Chemin JY, Desjardins B, Gallagher I, Grenier E (2000) M2AN Math. Model. Numer. Anal. 34:315-335

10. Clark R, Ferziger J, Reynolds W (1979) J. Fluid Mech. 91:1-16

11. Constantin P, Foias C (1988) Navier-Stokes equations. Univ. of Chicago Press, Chicago

12. Deville M, Fischer P, Mund E (2002) High-order methods for incompressible fluid flow. Cambridge Univ. Press, Cambridge

13. Fan J, Ozawa T (2009) Nonlinearity 22:553-568

14. Fischer P (1997) J. Comp. Phys. 133:84-101

15. Fischer P, Mullen J (2001) C. R. Acad. Sci. Paris Sér. I Math. 332:265-270

16. Galdi GP, Layton WJ (2000) Math. Models Methods Appl. Sci. 10:343-350

17. Geurts BJ, Holm DD (2003) Phys. Fluids 15:L13-L16

18. Härtel C, Meiburg E, Necker F (2000) J. Fluid Mech. 418:189-212

19. Ishimura N, Morimoto H (1999) Math. Models Methods Appl. Sci. 9:1323-1332

20. Layton WJ, Lewandowski R (2008) Discrete Contin. Dyn. Syst. Ser. B 6:111-128

21. Leonard A (1974) Adv. in Geophysics 18A:237-248

22. Maday Y, Patera AT (1989) Spectral element methods for the incompressible Navier-Stokes equations. In: Noor AK and Oden JT (eds) State-of-the-Art Surveys in Computational Mechanics, ASME, N.Y.

23. Nerli A, Camarri S (2006) Meccanica 41:671-680

24. Özgökmen T, Iliescu T, Fischer P (2009) Ocean Modelling 26:134-155

25. Özgökmen T, Iliescu T, Fischer P (2009) Ocean Modelling, accepted.

26. Özgökmen T, Iliescu T, Fischer P, Srinivasan A, Duan J (2007) Ocean Modelling 16:106-140

27. Patera A (1984) J. Comput. Phys. 54:468-488

28. Pope S (2000) Turbulent flows. Cambridge Univ. Press, Cambridge

29. Rummler B (1997) Z. Angew. Math. Mech. 77:669-675

30. Stolz S, Adams NA (1999) Phys. Fluids 11:1699-1701

31. Vreman B, Geurts B, Kuerten H (1996) Theor. Comput. Fluid Dyn. 8:309-324

32. Vreman B, Geurts B, Kuerten H (1997) J. Fluid Mech. 339:357-390

33. Winckelmans GS, Jeanmart H, Carati D (2002) Phys. Fluids 14:1809-1811

34. Winckelmans GS, Wray AA, Vasilyev OV, Jeanmart H. (2001) Phys. Fluids 13:1385-1403 